\title{
Propiedades psicométricas de la Escala del Síndrome de Dependencia de la Nicotina (NDSS) en una muestra de fumadores que solicitan tratamiento por su dependencia del alcohol
}

\section{Psychometric properties of the Nicotine Dependence Syndrome Scale (NDSS) in a sample of smokers treated for their alcohol dependence}

\author{
Elisardo Becoña*; Luis Nogueiras**; Gerardo \\ FlóReZ ${ }^{\star *}$; SANDRA ÁlVAREZ ${ }^{\star *}$; Dolores VÁzQueZ * ${ }^{* *}$
}

\author{
* Unidad de Tabaquismo. Universidad de Santiago de Compostela. \\ Facultad de Psicología. Departamento de Psicología Clínica y \\ Psicobiología. Santiago de Compostela. \\ ** Unidad de Conductas Adictivas, Complejo Hospitalario de \\ Ourense. \\ Enviar correspondencia a: \\ Elisardo Becoña. Unidad de Tabaquismo. Universidad de Santiago \\ de Compostela. Facultad de Psicología. Departamento de \\ Psicología Clínica y Psicobiología. Campus sur. 15782 Santiago \\ de Compostela. E-mail: elisardo.becona@usc.es.
}

\section{RESUMEN}

La evaluación de la dependencia de la nicotina con instrumentos breves es de gran relevancia para una mejor detección de este trastorno. En el presente estudio se presentan los resultados con la Escala del Sindrome de Dependencia de la Nicotina (Nicotine Dependence Syndrome Scale, NDSS) de Shiffman, Waters y Hickcox (2004) en una muestra de 183 pacientes que demandaron tratamiento en una Unidad de Alcoholismo y que eran además fumadores de cigarrillos. Los resultados indican que el factor general que evalúa dependencia de la nicotina (NDSS-T) tiene una buena fiabilidad (coeficiente alfa de Cronbach $=0.80$ ). El análisis factorial indica la existencia en esta muestra de cuatro de los cinco factores propuestos en la versión original: impulso, prioridad, continuidad y estereotipia. La fiabilidad de las escalas derivadas factorialmente oscilan de muy buenas (0.80) a moderadas (0.63). La NDSS-T correlaciona significativamente con el Cuestionario de Tolerancia de Fagerström (FTO), con los criterios de dependencia de la nicotina del DSM-IV evaluados con la entrevista SCID y con el número de cigarrillos fumados diariamente. Las curvas ROC indican que la NDSS-T tiene una puntuación de 0.80 bajo la curva (0.70 para el FT0), lo que indica que predice adecuadamente la dependencia de la nicotina. Este estudio confirma la utilidad de este nuevo instrumento para evaluar la dependencia de la nicotina en los fumadores que al mismo tiempo abusan o dependen del alcohol.

Palabras clave: Dependencia, evaluación, NDSS, nicotina, alcohol, fumadores. .

\section{ABSTRACT}

The assessment of nicotine dependence with brief instruments is of great relevance for the better detection of this disorder. Here we present the results with the Nicotine Dependence Syndrome Scale (NDSS) by Shiffman, Waters and Hickcox (2004) in a sample of 183 patients treated at an Alcohol Dependence Unit who were also cigarette smokers. The results indicate that the general factor which evaluates nicotine dependence (NDSS-T) has good reliability (Cronbach's alpha $=0.80$ ). Factor analysis identifies four of the five factors proposed in the original version, those of drive, priority, continuity and stereotypy. Reliability of the scales derived ranges from very good $(0.80)$ to moderate (0.63). The NDSS-T correlates significantly with the Fagerström Tolerance Questionnaire (FTO), with the DSM-IV criteria for nicotine dependence assessed through the SCID, and with the number of cigarettes smoked per day. The ROC curves indicate an NDSS-T score of 0.80 under the curve ( 0.70 for the FTND), showing that it adequately predicts nicotine dependence. This study confirms the utility of this new instrument for assessing nicotine dependence in smokers who also abuse or depend upon alcohol.

Key words: Dependence, assessment, NDSS, nicotine, alcohol, smokers. 


\section{INTRODUCCIÓN}

S e hipotetiza que la dependencia de la nicotina es un proceso central que subyace al motivo por el que una persona mantiene la conducta de fumar y por el que experimenta una gran dificultad en dejar de fumar cuando lo intenta. Aunque la mayoría de los fumadores son dependientes de la nicotina, no todos lo son, ni cumplen criterios de dependencia según el DSM-IV (Dierker, 2007; Donny y Dierker, 2007; Shiffman y Paton, 1999). La nicotina es una droga con características especiales: es la más consumida después del café, su poder adictivo es enorme y es la responsable de producir la mayor mortalidad evitable hoy conocida en las sociedades desarrolladas (U.S.D.H.H.S., 2004).

El poder adictivo de la nicotina explica porqué muchos fumadores que quieren dejar de fumar fracasan una y otra vez y no lo consiguen (Pereiro, Becoña, Córdoba, Martínez y Pinet, 2007). Su poder adictivo se ha ido demostrando a lo largo de miles de estudios en estas pasadas décadas (ver U.S.D.H.H.S., 1988; 2000). La fuerza de la dependencia de la nicotina producida por el consumo de cigarrillos se debe a varios factores (Becoña, 2006): 1) la nicotina produce múltiples efectos de reforzamiento positivo (ej., mejora la concentración, el estado de ánimo, disminuye la ira y el peso); 2) después de una inhalación la nicotina tarda menos de 10 segundos en llegar al cerebro, produciéndose un efecto de la sustancia casi instantáneo a su ingestión; 3) el fumador puede modular, por la forma en que fuma el cigarrillo, la dosis de nicotina que necesita; 4 ) la conducta de fumar se realiza repetidamente (por ejemplo, un fumador de un paquete de cigarrillos se administra nicotina aproximadamente unas 200 veces al día); y, 5) múltiples señales, previamente asociadas a la conducta de fumar por procesos de condicionamiento, elicitan dicha conducta.

Clinicamente, para saber si una persona tiene dependencia de la nicotina utilizamos los criterios diagnósticos de la dependencia de una droga, según el DSM-IV-TR (American Psychiatric Association, 2000) o la CIE-10 (Organización Mundial de la Salud, 1992). También podemos aplicar dichos criterios para el sindrome de abstinencia de la nicotina, que tiene unos sintomas característicos para esta sustancia, igualmente basados en el DSM-IV-TR o la CIE-10. Los síntomas del sindrome de abstinencia comienzan al cabo de unas pocas horas (2-12 horas) y alcanzan su punto álgido a las 24-48 horas de dejar de fumar. La mayoría de los síntomas duran un promedio de cuatro semanas, pero la sensación de necesidad de nicotina (craving) puede durar 6 meses o más. Dichos sintomas varian de uno a otro fumador.

Cuantificar el grado de dependencia de la nicotina de un fumador es un aspecto importante ya que aquellos que tienen una dependencia elevada suelen precisar una terapia más intensiva (Fiore, Jaen, Baker, Balley, Benowitz, Curry, et al., 2008). En personas con dependencia del alcohol sabemos que el consumo y la dependencia de la nicotina es mayor que la de los fumadores de la población general (Nieva, Gual, Ortega y Mondón, 2004). Sin embargo, los profesionales de las Unidades de Alcoholismo no siempre les ofrecen ayuda para dejar de fumar (Ballbé, Mondón, Nieva, Walter, Saltó y
Gual., 2008) junto al tratamiento que se les aplica para que dejen de beber (Nieva y Gual, 2005). Por ello es necesario disponer de datos sobre estas personas cuando nos demandan tratamiento y conocer más en profundidad sus caracteristicas para poder ayudarles. Aun cuando muchos pacientes con el paso del tiempo dejan de beber y de fumar sin ayuda (Carballo, Fernández-Hermida, Secades-Villa y Garcia-Rodríguez, 2008) también sabemos que cuando se ponen en práctica tratamientos en personas dependientes del alcohol para ayudarles a dejar de fumar se obtienen resultados superiores a la no intervención (Nieva et al., 2004).

Actualmente disponemos de varias medidas para valorar el grado de dependencia de la nicotina (Becoña y Lorenzo, 2004). Las que han demostrado tener más utilidad para el tratamiento son los criterios diagnósticos del DSM-IV-TR y el Test de Dependencia de la Nicotina de Fagerström (FTND, Heatherton, Kozlowski, Frecker y Fagerström, 1991). Este test consta de sólo 6 preguntas que nos permite evaluar el nivel de dependencia del fumador. Es el instrumento de evaluación de la dependencia más utilizado. Sin embargo su fiabilidad es reducida, habitualmente no superior a 0.60 , como ocurre en España (ej., Becoña y Vázquez, 1988) y en distintos estudios realizados en otros paises (Shadel et al., 2000), lo que limita su utilidad diagnóstica y predictiva, a pesar de que su estructura factorial es consistente (de Leon, Becoña, Gurpegui, González-Pinto y Díaz, 2002). Debido a su baja fiabilidad en los últimos años se ha sugerido evaluar la dependencia de la nicotina con escalas más completas y que se adecuen más estrictamente a los criterios del DSM-IV-TR. Esto es debido a que cuando se elaboró la escala de Fagerström los criterios para evaluar la dependencia de la nicotina eran distintos a los actuales. Por ello, suele encontrarse actualmente una baja concordancia entre el diagnóstico de dependencia de la nicotina con el DSM-IV-TR y la escala de Fagerström (Moolchan et al., 2002).

Una de las escalas que parece ser útil para evaluar la dependencia, en la línea de los criterios del DSM-IV-TR, es la Escala del Sindrome de Dependencia de la Nicotina (NDSS, Nicotine Dependence Syndrome Scale) de Shiffman, Waters y Hickcox (2004). La misma consta en su última versión de 19 ítems. Es, por tanto, una escala corta que permite evaluar un factor general de dependencia de la nicotina. Para la elaboración de dicho instrumento sus autores partieron del concepto de sindrome de dependencia de Edwards (1976), a partir del cual suele conceptualizarse hoy la dependencia de sustancias psicoactivas. También parten del mismo los actuales sistemas clasificatorios asumiendo que la dependencia es multifacética. Por ello, la NDSS evalúa cinco aspectos que considera centrales para entender la dependencia de la nicotina, como son (indicamos entre paréntesis algunos de los ítems como los evalúa la NDSS):

I. Impulso (drive), que captura el craving y el sindrome de abstinencia (ej., item 18. Después de estar un tiempo sin fumar, necesito hacerlo para aliviar las sensaciones de inquietud e irritabilidad) y el sentido subjetivo de la compulsión a fumar (ej., item 14. Cuando realmente deseo un cigarrillo, parece que 
estoy bajo el control de alguna fuerza desconocida que no puedo dominar).

II. Prioridad, que refleja la preferencia conductual de fumar por encima de otros reforzadores (ej., item 5. Tiendo a evitar los restaurantes donde no se permite fumar, incluso aunque me guste su comida).

III. Tolerancia, que evalúa la reducción de la sensibilidad a los efectos de fumar (ej., item 8. Comparado con cuando empecé a fumar, necesito fumar mucho más ahora para conseguir el mismo efecto).

IV. Continuidad, que se refiere a la regularidad de fumar (ej., item 1. Mi forma de fumar es muy irregular a lo largo del día. No es extraño que fume muchos cigarrillos durante una hora y luego no fume ninguno hasta horas después).

V. Estereotipia, que evalúa la invarianza o monotonía de fumar (ej., ítem 2. Mi forma de fumar no se ve afectada por otras cosas. Fumo más o menos lo mismo si estoy relajado o si estoy trabajando, contento o triste, solo o en compañía de otros, etc.).

Los anteriores aspectos son centrales en la dependencia de la nicotina. Por ello, la NDSS evaluaría mejor el síndrome de dependencia que el FTND de Fagerström. Además, tendría la ventaja de evaluar varias facetas (las cinco propuestas) más que constituir una medida unidimensional de la dependencia.

Los primeros estudios de Shiffman et al. (2004) con la NDSS confirman los cinco factores o dimensiones propuestas y la utilidad de la escala, mostrando la misma tanto validez concurrente como predictiva. Utilizaron para ello distintas muestras, habitualmente de fumadores de 10 o más cigarrillos diarios o de fumadores dependientes de la nicotina. En dichos estudios se confirmó la estructura factorial de cinco factores y una adecuada fiabilidad para la escala total y las subescalas. Estudios posteriores (ej., Shiffman y Sayette, 2005) examinaron la utilidad de la NDSS en fumadores ocasionales y regulares, en fumadores adolescentes, de 12 a 18 años (Clark, Wood, Martin, Cornelius, Lynch y Shiffman, 2005) y en fumadores universitarios (Costello, Dierker, Sledjeski, Flaherty, Flay y Shiffman, 2007; Sledjeski, Dierker, Costello, Shiffman, Dony y Flay, 2007). En todos ellos suelen confirmarse los factores originales propuestos por Shiffman et al. (2004). Por ejemplo en el estudio de Clark et al., (2005) todas las escalas de la NDSS correlacionan con las puntuaciones del FTND y predicen la cantidad fumada por estas personas en el seguimiento de un año.

La NDSS también se ha utilizado en fumadores afroamericanos que fumaban pocos cigarrillos (Okuyemi, Pulvers, Cox, Thomas, Kaur, Mayo, et al, 2007) y en fumadores con distintos niveles de consumo de cigarrillos (Piper et al., 2008), analizando su validez convergente y discriminante en relación a otros cuestionarios (Courvoisier y Etter, 2008). También la NDSS se ha adaptado a otras lenguas y países (ej., Broms, Madden, Health, Pergadia, Shiffman y Kaprio, 2007). Todo ello muestra el interés que hay a nivel investigador y clínico desde que ha aparecido la NDSS, motivado en parte por la adecuación de sus propiedades psicométricas.

El objetivo del presente estudio es evaluar las propiedades psicométricas de la Escala del Síndrome de Dependencia de la Nicotina (NDSS) en una muestra de fumadores que solicitan tratamiento en una Unidad de Alcoholismo, en su mayoría por abuso o dependencia del alcohol, para ver su utilidad y poder discriminativo, utilizando para ello distintas escalas que evalúan dependencia de la nicotina junto a los criterios de dependencia de la nicotina del DSM-IV-TR.

\section{MÉTODO}

\section{Participantes}

La muestra fue obtenida en la Unidad de Conductas Adictivas de Ourense de entre aquellos pacientes que hicieron una demanda por abuso o dependencia del alcohol, fundamentalmente, incluyéndose también algunos casos por demanda de juego patológico y algunos específicos por tabaco. El criterio de inclusión de las personas en el presente estudio era que demandasen tratamiento en la Unidad por adicción al alcohol, juego patológico y tabaco y que fuesen fumadoras.

La muestra total está formada por 183 fumadores evaluados en dicha Unidad para realizar tratamiento en la misma. La edad media de esta muestra fue de 42.28 años (D. T. = 11.32), rango de 17 a 69 años. De ellos, 155 (84.7\%) eran varones y $28(15.3 \%)$ mujeres.

El Comité de Bioética de la Universidad de Santiago de Compostela autorizó la realización de este estudio.

\section{Cuestionario}

Además de la evaluación de las variables clínicas por las que demandaban tratamiento, se recogieron datos sobre variables sociodemográficas, de consumo y de historia de fumador. Igualmente se utilizaron los siguientes instrumentos de evaluación:

- Criterios de dependencia de la nicotina según criterios DSM-IV-TR. Se utilizó la Structured Clinical Interview for DSM-IV (SCID-IV, First, Spitzer, Gibbson y Williams (1998), entrevista diagnóstica semiestructurada compuesta por tantos módulos diagnósticos como categorías diagnósticas tiene el DSM y que permite establecer de forma clara y precisa la existencia de un trastorno.

Dado que la SCID no contempla la entrevista para la dependencia de la nicotina, elaboramos la entrevista diagnóstica correspondiente (Becoña, López, Fernández del Río y Míguez, 2008), basándonos en la evaluación de la dependencia de otras sustancias (concretamente cocaína, ver López, Becoña, Lloves, Moneo, Vieitez, Cancelo, et al., 2007) y en los criterios diagnósticos para la dependencia de la nicotina del DSM-IV (American Psychiatric Association, 2000) (ver tabla 1). 
Tabla 1. Criterios de la SCID utilizados para evaluar dependencia de la nicotina.

\begin{tabular}{|c|c|}
\hline Dependencia de sustancias, criterios DSM-IV-TR & SCID-nicotina \\
\hline $\begin{array}{l}\text { 1.TOLERANCIA definida por: } \\
\text { a. Necesidad de cantidades marcadamente crecientes } \\
\text { para conseguir la intoxicación o el efecto deseado. } \\
\text { b. El efecto de la misma cantidad de sustancia dis- } \\
\text { minuye con su consumo continuado. }\end{array}$ & $\begin{array}{l}\text { 6. ¿Ha comprobado que cada vez tenia que fumar más cantidad de cigarrillos para conseguir las sensaciones que } \\
\text { queria, en comparación con lo que fumaba cuando empezó a fumar? } \\
\text { SI } \square \text { ¿Cuántos cigarrillos más? } \\
\text { NO } \square \text { ¿Ha comprobado que cuando consumia la misma cantidad que al principio obtenia un efecto menor que } \\
\text { antes? } \\
\text { SI } \square \text { NO } \square \\
\text { ¿O que si fumara al principio la misma cantidad que ahora se intoxicaria? SI } \square \text { NO } \square\end{array}$ \\
\hline $\begin{array}{l}\text { 2.ABSTINENCIA definida por cualquiera de los siguientes } \\
\text { items: } \\
\text { a. Sindrome de abstinencia caracteristico de la sustan- } \\
\text { cia. } \\
\text { b. Se toma la sustancia o similar para aliviar o evitar los } \\
\text { sintomas de abstinencia. }\end{array}$ & $\begin{array}{l}\text { 7. ¿Ha experimentado alguna vez sintomas de abstinencia cuando reducia o interrumpia el consumo de tabaco? } \\
\text { NO } \square \\
\text { SI } \square \text { ¿Qué sintomas tenia? ( } 4 \text { o más de los siguientes sintomas): Estado de ánimo disfórico o depresivo, } \\
\text { irritabilidad, ansiedad, inquietud o impaciencia, aumento del apetito o aumento del peso, insomnio, frustración o } \\
\text { ira, dificultades de concentración y disminución de la frecuencia cardiaca. } \\
\text { Si tuvo sintomas de abstinencia: Después de estar sin fumar durante unas horas o más, ¿a menudo tenía que } \\
\text { volver a hacerlo para evitar los sintomas de abstinencia? SI } \square \text { NO } \square \\
\text { ¿Y tomar chicles de nicotina o usar parches de nicotina para evitar esos sintomas? SI } \square \text { NO } \square \\
\text { ¿Y tomar otros fármacos para dejar de fumar? NO } \square \mathrm{SI} \square \text { ¿Cuáles? }\end{array}$ \\
\hline $\begin{array}{l}\text { 3. La sustancia se toma con frecuencia en cantidades } \\
\text { mayores o durante un periodo más largo de lo que se pre- } \\
\text { tendia. }\end{array}$ & $\begin{array}{l}\text { 1. ¿A menudo comprueba que cuando empieza a fumar acaba fumando más cigarrillos de los que tenía pensado? } \\
\text { SI } \sqcup \\
\text { NO } \square \text { ¿Y fumar durante más tiempo de lo que tenía previsto? (ej., "en año nuevo lo dejo"): SI } \square \text { NO } \square\end{array}$ \\
\hline $\begin{array}{l}\text { 4. Deseo persistente o esfuerzos infructuosos de controlar } \\
\text { o interrumpir el consumo. }\end{array}$ & $\begin{array}{l}\text { 2. ¿Ha intentado controlar su consumo de tabaco o dejar de fumar? } \\
\text { SI } \sqcup \text { ¿Ha dejado de fumar del todo en alguna ocasión? SI } \sqcup \text { NO } \sqcup \\
\text { NO } \square \text { ¿Quiere dejar de fumar o reducir el consumo? } \quad \text { SI } \square \text { NO } \square\end{array}$ \\
\hline $\begin{array}{l}\text { 5. Emplea mucho tiempo en actividades de obtención de la } \\
\text { sustancia, en el consumo o en la recuperación de los efec- } \\
\text { tos de la sustancia. }\end{array}$ & $\begin{array}{l}\text { 3. ¿Ha empleado mucho tiempo de su vida en fumar o haciendo lo necesario para conseguir tabaco? (ej., salir de } \\
\text { casa por la noche porque se quedó sin tabaco). SI } \square \text { NO } \square \\
\text { ¿Le lleva mucho tiempo volver a su estado normal después de fumar? SI } \square \text { NO } \square\end{array}$ \\
\hline $\begin{array}{l}\text { 6. Reducción de importantes actividades sociales, laborales } \\
\text { o recreativas debido al consumo. }\end{array}$ & $\begin{array}{l}\text { 4. ¿Ha habido periodos en los que fumaba con tanta frecuencia que perdia mucho tiempo de su trabajo, o dejaba } \\
\text { de estar con amigos o familiares porque no podia fumar con ellos, o dejaba de ir a lugares como el cine, museos, } \\
\text { restaurantes ... porque no se podia fumar? } \\
\text { SI } \square \text { NO } \square\end{array}$ \\
\hline $\begin{array}{l}\text { 7. Continúa tomando la sustancia a pesar de tener con- } \\
\text { ciencia de problemas psicológicos o físicos, recidivantes o } \\
\text { persistentes, causados o exacerbados por el consumo de la } \\
\text { sustancia. }\end{array}$ & $\begin{array}{l}5 . \text { ¿El consumo de tabaco le ha causado problemas psicológicos tales como sentirse deprimido, ansioso o le ha } \\
\text { causado problemas para dormir? SI } \sqcup \mathrm{NO} \sqcup \\
\text { Si no se conoce: ¿Le ha causado problemas físicos significativos o ha agravado otros que ya tenía? SI } \square \text { NO } \square \\
\text { Si contesta afirmativamente a alguna de las dos: ¿A pesar de todo siguió fumando? } \quad \text { SI } \square \text { NO } \square\end{array}$ \\
\hline
\end{tabular}

Fuente: Becoña, López, Fernández del Rio y Miguez (2008).

Tabla 2. Versión española de la Escala del síndrome de dependencia de la nicotina (NDSS) de Shiffman et al. (2004).

Marque una $\mathrm{X}$ en la alternativa que mejor describa cada una de las siguientes afirmaciones:

\begin{tabular}{|c|c|c|c|c|c|}
\hline & No es cierto & $\begin{array}{l}\text { Algo } \\
\text { cierto }\end{array}$ & $\begin{array}{l}\text { Moderadamente } \\
\text { cierto }\end{array}$ & $\begin{array}{l}\text { Muy } \\
\text { cierto }\end{array}$ & $\begin{array}{l}\text { Totalmente } \\
\text { Cierto }\end{array}$ \\
\hline \multicolumn{6}{|l|}{ 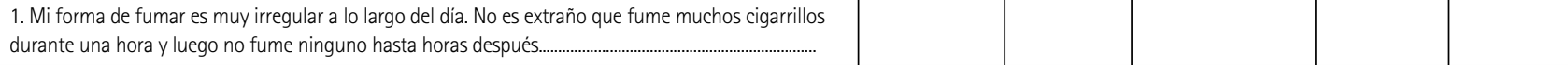 } \\
\hline \multicolumn{6}{|l|}{$\begin{array}{l}\text { 2. Mi forma de fumar no se ve muy afectada por otras cosas. Fumo más o menos lo mismo si } \\
\text { estoy relajado o si estoy trabajando, contento o triste, solo o en compañia de otros, etc.................... }\end{array}$} \\
\hline \multicolumn{6}{|l|}{$\begin{array}{l}\text { 3. Si tengo que hacer un viaje largo no me planteo viajar en avión porque sé que no está permitido } \\
\text { fumarar }\end{array}$} \\
\hline \multicolumn{6}{|l|}{ 4. A veces dejo de visitar a mis amigos no fumadores porque sé que me sentiré incómodo si fumo } \\
\hline \multicolumn{6}{|l|}{ 5. Tiendo a evitar los restaurantes donde no se permite fumar, incluso aunque me guste su comida } \\
\hline \multicolumn{6}{|l|}{ 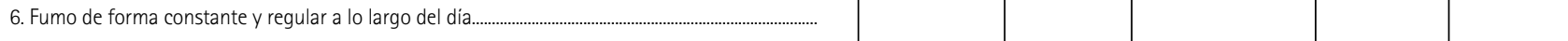 } \\
\hline \multicolumn{6}{|l|}{ 7. Fumo distinta cantidad de cigarrillos en función de la situación en la que esté } \\
\hline \multicolumn{6}{|l|}{$\begin{array}{l}\text { 8. Comparado con cuando empecé a fumar, necesito fumar mucho más ahora para conseguir el } \\
\text { mismo efecto. }\end{array}$} \\
\hline \multicolumn{6}{|l|}{$\begin{array}{l}\text { 9. Comparado con cuando empecé a fumar, ahora puedo fumar mucho más sin llegar a sentir } \\
\text { náuseas o malestar. }\end{array}$} \\
\hline 10. Tras pasar un tiempo sin fumar, necesito hacerlo para no sentirme mal............................................ & & & & & \\
\hline
\end{tabular}




\begin{tabular}{|c|c|c|c|c|c|}
\hline & No es cierto & $\begin{array}{l}\text { Algo } \\
\text { cierto }\end{array}$ & $\begin{array}{l}\text { Moderadamente } \\
\text { cierto }\end{array}$ & $\begin{array}{l}\text { Muy } \\
\text { cierto }\end{array}$ & $\begin{array}{l}\text { Totalmente } \\
\text { Cierto }\end{array}$ \\
\hline \multicolumn{6}{|l|}{ 11. Es dificil saber cuántos cigarrillos fumo al dia porque el número suele variar .................................... } \\
\hline \multicolumn{6}{|l|}{ 12. Tengo sensación de control sobre el tabaco. Puedo cogerlo o dejarlo en cualquier momento... } \\
\hline \multicolumn{6}{|l|}{$\begin{array}{l}\text { 13. El número de cigarrillos que fumo al dia varía según distintos factores: cómo me siento, qué } \\
\text { estoy haciendo, etc. }\end{array}$} \\
\hline \multicolumn{6}{|l|}{ 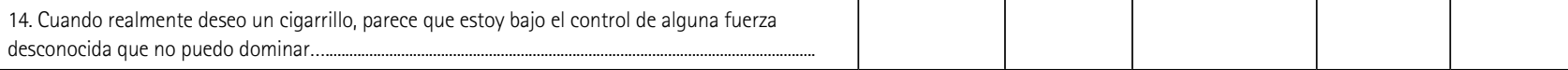 } \\
\hline \multicolumn{6}{|l|}{$\begin{array}{l}\text { 15. Desde que me he convertido en un fumador habitual, la cantidad que fumo ha sido la misma o } \\
\text { ha disminuido un poco }\end{array}$} \\
\hline \multicolumn{6}{|l|}{$\begin{array}{l}\text { 16. Siempre que estoy sin fumar durante algunas horas, siento unas ganas muy fuertes de } \\
\text { hacerlo }\end{array}$} \\
\hline \multicolumn{6}{|l|}{ 17. Mi consumo de cigarrillos es bastante regular a lo largo del dia........................................................ } \\
\hline \multicolumn{6}{|l|}{$\begin{array}{l}\text { 18. Después de estar un tiempo sin fumar, necesito hacerlo para aliviar las sensaciones de inquietud } \\
\text { e irritabilidad. }\end{array}$} \\
\hline 19. Fumo la misma cantidad de tabaco durante la semana que en el fin de semana....................................... & & & & & \\
\hline
\end{tabular}

- Escala del Síndrome de Dependencia de la Nicotina de Shiffman et al. (2004). Esta escala consta de 19 items y permite evaluar un factor general de dependencia de la nicotina y varios factores derivados de la misma. En la validación original aparecen los factores de impulso, prioridad, tolerancia, continuidad y estereotipia.

La versión utilizada de la NDSS en castellano para el presente estudio se obtuvo a partir del cuestionario de 19 ítems de Shifman et al. (1994) mediante el procedimiento de la traducción inversa (back translation) (Brislin, Lonner y Thorndike, 1973). Tres personas distintas hicieron la traducción del inglés al castellano. Otras tres personas, independientes de las anteriores, expertas en el tema de tabaquismo y adicciones, revisaron la traducción y consensuaron una sola versión de la escala. Finalmente, dos traductores tradujeron la versión española a la inglesa para comprobar que la española equivalía a la inglesa, como asi fue. Las opciones de respuesta para cada uno de los ítems son cinco (no es cierto, algo cierto, moderadamente cierto, muy cierto, totalmente cierto), otorgándose la puntuación 1 a la primera alternativa y 5 a la última. La versión española de la NDSS puede verse en la tabla 2.

- Cuestionario de Tolerancia (FT0) de Fagerström (1978). Este cuestionario consta de 8 items y evalúa la dependencia de la nicotina. Se ha utilizado la versión española de Becoña, Gómez-Durán, Alvarez-Soto y García (1992). En una revisión comprensiva de estudios que utilizaron este cuestionario se encontró que 14 de las 16 diferentes clases de datos, relacionando marcadores bioquímicos con el cuestionario, tenian correlaciones significativas (Pomerleau, Carton, Lutzke, Flessland y Pomerleau, 1994). La puntuación oscila de 0 a 11 con un punto de corte de 7 para la dependencia de la nicotina (Fagerström y Schneider, 1989). Su fiabilidad en la versión española es adecuada, concretamente de 0.66 .

\section{Análisis estadístico}

Se utilizaron distintos tipos de análisis estadístico, como análisis factorial, comparación de medias mediante pruebas $t$, análisis de varianza para más de dos grupos, análisis de correlaciones entre las escalas, curvas ROC, etc., utilizando el paquete estadístico SPSS v. 15.0.

\section{Procedimiento}

A todos los pacientes que demandaron tratamiento se les solicitó autorización para participar en el estudio una vez que se les hubo explicado en que consistía el mismo. El cuestionario se cumplimentó junto a otros relacionados directamente con la demanda de su tratamiento.

\section{RESULTADOS}

\section{Análisis factorial de la NDSS}

Con la muestra utilizada $(\mathrm{N}=183)$ la medida de adecuación muestral de Kaiser-Meyer-Olkin es buena (0.801) para proceder a realizar un análisis factorial. El análisis factorial mediante componentes principales indica que el número de factores que tienen una comunalidad superior a 1 es de 6. Sin embargo, el análisis de sedimentación indica que el número ideal de factores a extraer sería de 4 (figura 1), que son los factores que hemos retenido para el análisis (tablas 3 y 4$)$.

Al rotar ortogonalmente los 4 primeros factores (rotación ortogonal varimax) se observa que éstos tienen una interpretación adecuada, y la misma se ajusta bastante bien a los resultados de Shiffman et al. (2004) con la validación de 
este cuestionario en muestras norteamericanas. Los cuatro factores obtenidos explican el 55.02\% de la varianza total (por factores el 25.50\%, 14.42\%, 8.12\% y 6.98, respectivamente). De este modo, los factores que se obtendrian serian perfectamente interpretables y serian los de (ver tabla 4), I-Impulso, II- Prioridad, III-Continuidad, y IV- Estereotipia. Estos factores son los que retenemos para los posteriores análisis. Hemos utilizado una puntuación en la carga factorial de 0.30 o más para retener los ítems en cada factor, tal como recomienda Kline (1994, p. 53). De este modo la primera escala tiene 9 ítems, la segunda 8 ítems, la tercera 4 items, y la cuarta 6 ítems. Para el primer factor general de dependencia (NDSS-T) hemos retenido como sugieren Shiffman et al. (2004) los ítems que saturan en el primer factor de la matriz factorial sin rotar con una carga superior a 0.30 .
De este modo la subescala general de dependencia queda constituída por 13 de los 19 ítems (tabla 3).

Para el análisis de los resultados a partir de las escalas derivadas factorialmente se han utilizado en los diferentes estudios realizados con la NDSS dos tipos de comparaciones, las basadas en las puntuaciones factoriales de cada factor (Shiffman et al., 2004), o en la suma de los ítems que han saturado significativamente en cada factor (Clark et al., 2005; Sterling et al., 2008). Siguiendo las sugerencias de Clark et al. (2005) hemos utilizado este último procedimiento, sumando el valor de los ítems significativos en cada factor, siempre que su saturación en el factor fuese 0.30 o más para tomarlo en consideración. Este procedimiento de cálculo es más sencillo y tiene eliminamos el inconveniente de que la utilización de las puntuaciones factoriales varian de una a otra muestra.

Tabla 3. Análisis factorial (componentes principales), matriz factorial sin rotar, 6 factores $(\mathrm{N}=183)$.

\begin{tabular}{|c|c|c|c|c|c|c|}
\hline & 1 & 2 & 3 & 4 & 5 & 6 \\
\hline $\begin{array}{l}\text { 1. Mi forma de fumar es muy irregular a lo largo del dia. No es extraño que fume muchos cigarrillos } \\
\text { durante una hora y luego no fume ninguno hasta horas después........................................................................ }\end{array}$ & & -.634 & & & & .332 \\
\hline $\begin{array}{l}\text { 2. Mi forma de fumar no se ve muy afectada por otras cosas. Fumo más o menos lo mismo si estoy } \\
\text { relajado o si estoy trabajando, contento o triste, solo o en compañia de otros, etc........................................ }\end{array}$ & & & & & .861 & \\
\hline $\begin{array}{l}\text { 3. Si tengo que hacer un viaje largo no me planteo viajar en avión porque sé que no está permitido } \\
\text { fumar. }\end{array}$ & .410 & & -.532 & .273 & & \\
\hline 4. A veces dejo de visitar a mis amigos no fumadores porque sé que me sentiré incómodo si fumo. & .417 & .283 & -.453 & .339 & & .275 \\
\hline $\begin{array}{l}\text { 5. Tiendo a evitar los restaurantes donde no se permite fumar, incluso aunque me guste su } \\
\text { comida. }\end{array}$ & .646 & & & & & \\
\hline 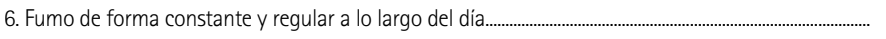 & & .738 & .358 & & & \\
\hline 7. Fumo distinta cantidad de cigarrillos en función de la situación en la que esté................................................ & .442 & -.369 & .429 & .282 & -.276 & -.252 \\
\hline $\begin{array}{l}\text { 8. Comparado con cuando empecé a fumar, necesito fumar mucho más ahora para conseguir el } \\
\text { mismo efecto }\end{array}$ & .666 & & & -.448 & & \\
\hline 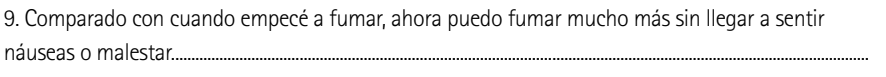 & .618 & & .341 & -.290 & & \\
\hline 10. Tras pasar un tiempo sin fumar, necesito hacerlo para no sentirme mal..................................................... & .750 & & & & & \\
\hline 11. Es dificil saber cuántos cigarrillos fumo al dia porque el número suele variar......................................... & .427 & -.449 & & & & -.250 \\
\hline 12. Tengo sensación de control sobre el tabaco. Puedo cogerlo o dejarlo en cualquier momento.......... & -.425 & & .424 & .287 & & \\
\hline 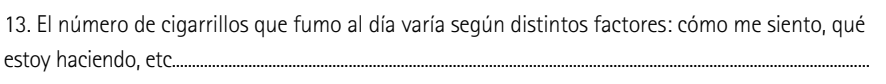 & .402 & -.524 & .344 & .392 & & \\
\hline 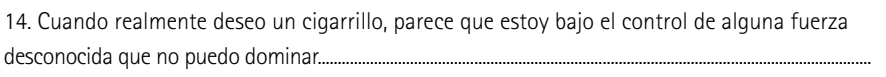 & .757 & & & & & \\
\hline $\begin{array}{l}\text { 15. Desde que me he convertido en un fumador habitual, la cantidad que fumo ha sido la misma o } \\
\text { ha disminuido un poco. }\end{array}$ & & .431 & & .587 & & -.294 \\
\hline 16. Siempre que estoy sin fumar durante algunas horas, siento unas ganas muy fuertes de hacerlo.. & .812 & & & & & \\
\hline 17. Mi consumo de cigarrillos es bastante regular a lo largo del dia........................................................................ & & .745 & .399 & & & \\
\hline 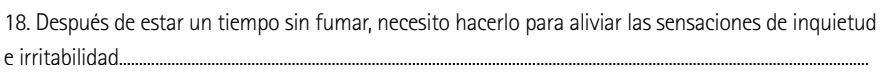 & .807 & & & & & \\
\hline 19. Fumo la misma cantidad de tabaco durante la semana que en el fin de semana.................................... & & .369 & & & .306 & .630 \\
\hline
\end{tabular}


Tabla 4. Análisis factorial (componentes principales), matriz factorial rotada (varimax), 4 factores $(\mathrm{N}=183)$.

\begin{tabular}{|c|c|c|c|c|}
\hline & 1 & 2 & 3 & 4 \\
\hline $\begin{array}{l}\text { 1. Mi forma de fumar es muy irregular a lo largo del dia. No es extraño que fume muchos cigarrillos durante una hora y luego no fume } \\
\text { ninguno hasta horas después..................................................................................... }\end{array}$ & & & .578 & -.332 \\
\hline $\begin{array}{l}\text { 2. Mi forma de fumar no se ve muy afectada por otras cosas. Fumo más o menos lo mismo si estoy relajado o si estoy trabajando, } \\
\text { contento o triste, solo o en compañía de otros, etc................................................. }\end{array}$ & & & & .294 \\
\hline 3. Si tengo que hacer un viaje largo no me planteo viajar en avión porque sé que no está permitido fumar................................................. & & .743 & & \\
\hline 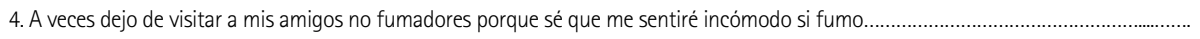 & & .750 & & \\
\hline 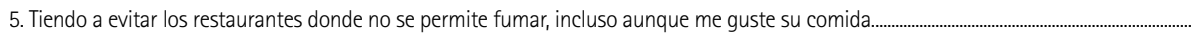 & .321 & .558 & & \\
\hline 6. Fumo de forma constante y regular a lo largo del dia........................... & .260 & & -.272 & .748 \\
\hline 7. Fumo distinta cantidad de cigarrillos en función de la situación en la que esté.................................. & .261 & & .722 & \\
\hline 8. Comparado con cuando empecé a fumar, necesito fumar mucho más ahora para conseguir el mismo efecto.................................................... & .833 & & & \\
\hline 9. Comparado con cuando empecé a fumar, ahora puedo fumar mucho más sin llegar a sentir náuseas o malestar........................................ & .725 & & & \\
\hline 10. Tras pasar un tiempo sin fumar, necesito hacerlo para no sentirme mal. & .605 & .445 & & \\
\hline 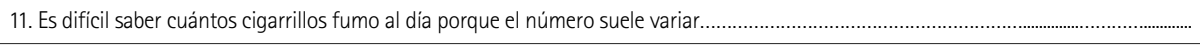 & & & .569 & \\
\hline 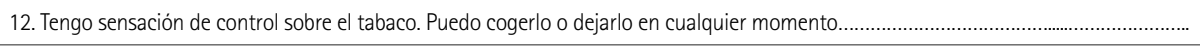 & -.381 & -.355 & & .429 \\
\hline 13. El número de cigarrillos que fumo al dia varia según distintos factores: cómo me siento, qué estoy haciendo, etc............................... & & & .826 & \\
\hline 14. Cuando realmente deseo un cigarrillo, parece que estoy bajo el control de alguna fuerza desconocida que no puedo dominar............. & .530 & .502 & & \\
\hline 15. Desde que me he convertido en un fumador habitual, la cantidad que fumo ha sido la misma o ha disminuido un poco...................... & -.487 & & & .590 \\
\hline 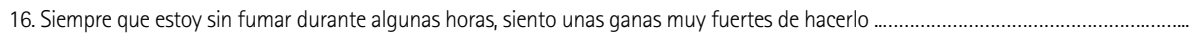 & .606 & .516 & & \\
\hline 17. Mi consumo de cigarrillos es bastante regular a lo largo del dia......... & & & -.263 & .793 \\
\hline 18. Después de estar un tiempo sin fumar, necesito hacerlo para aliviar las sensaciones de inquietud e irritabilidad.................................... & .585 & .574 & & \\
\hline 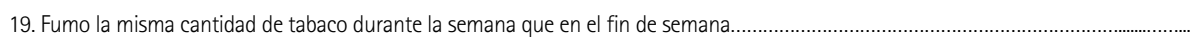 & & & & .345 \\
\hline
\end{tabular}

Factores: I-Impulso, II- Prioridad, III-Continuidad y IV- Estereotipia.

Tabla 5. Fiabilidad de las escalas derivadas factorialmente y de la escala total (NDSS-T).

\begin{tabular}{lcc}
\hline Escala & No items & Fiabilidad (coeficiente alfa) \\
\hline Impulso & 9 & .84 \\
Prioridad & 8 & .84 \\
Continuidad & 4 & .68 \\
Estereotipia & 6 & .63 \\
NDSS-T & 13 & .80 \\
\hline
\end{tabular}

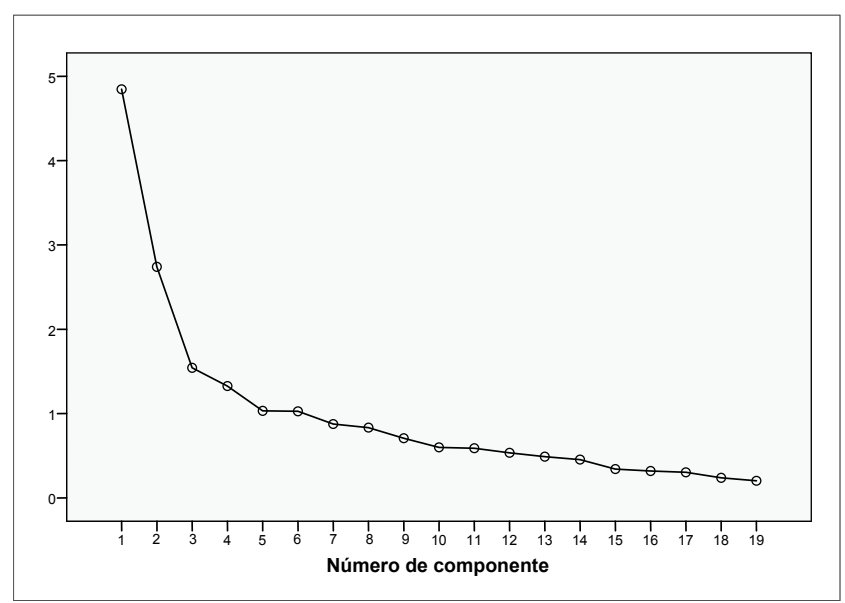

Figura 1. Gráfico e sedimentación.

\section{Fiabilidad de las escalas de la NDSS}

Hemos evaluado la fiabilidad, o consistencia interna, de las escalas mediante el coeficiente alfa de Cronbach para la escala total (NDSS-T) y para cada una de las escalas derivadas factorialmente. Los resultados indican una buena fiabilidad para la escala total, de 0.80, que está formada por 13 items del primer factor sin rotar. La fiabilidad de las escalas impulso y prioridad es buena (0.84 en ambas), y la de las escalas de continuidad y estereotipia es aceptable (0.68 y 0.63 respectivamente).

El análisis de la fiabilidad por ítems en cada una de las escalas confirma los resultados anteriores. La escala total tiene muy buena fiabilidad en cada item que la compone (de 0.77 a 0.80 ), así como el factor de impulso (de 0.80 a 0.86 ) y de prioridad (de 0.79 a 0.84). En el caso de las escalas de continuidad y de estereotipia la fiabilidad oscila entre 0.52 y 0.68 en el primer caso, y entre 0.51 y 0.66 en el segundo, lo que indica una fiabilidad adecuada.

\section{Validez concurrente}

La validez concurrente consiste en evaluar el mismo constructo con distintos métodos. En nuestro caso evaluamos la dependencia de la nicotina con criterios DSM-IV mediante la SCID y también con el Cuestionario de Tolerancia 
de Fagerström (FTQ) y la NDSS. Para ello utilizamos análisis de correlaciones entre ellas y también con el consumo diario de cigarrillos (tabla 6).

Los resultados indican que la correlación entre la NDSST y el FTO y SCID es alta y significativa: de $0.54(p<.001)$ entre NDSS-T y FTQ, y de 0.52 ( $p<.001)$ entre NDSS-T y SCID. Además, la correlación entre la escala impulso y el FTQ y SCID es estadísticamente significativa, de $0.60(p<.001)$ entre impulso y FTQ, y de $0.54(p<.001)$ entre impulso y SCID. Respecto a la escala de prioridad, su correlación con el FTO y SCID también es significativa $(r=0.53$ y $r=0.50 p<$ .001 , respectivamente), aunque menor que la que presentaba la escala impulso. Las escalas de continuidad y de estereotipia también correlacionan significativamente con el FTQ $(r=$ 0.15 y -0.14 respectivamente, $p<.05)$ y $\operatorname{SCID}(r=0.25, p<$ .001 , y $r=-0.18, p<.05$ respectivamente).

A su vez la correlación entre el FTO y SCID es de 0.33 $(p<.001)$. La puntuación en la NDSS-T correlaciona significativamente además con el número de cigarrillos fumados diariamente $(r=0.42, p<.001)$.

Un análisis más minucioso de cada uno de los ítems de la NDSS indica que casi todos sus 19 items correlacionan significativamente con la NDSS-T, el FTO, la SCID y el número de cigarrillos fumados diariamente (tabla 7).

Al utilizar los criterios de la SCID de dependiente (3 o más criterios) o no dependiente ( $0-2$ criterios) o del FTQ (7 o más puntos, dependiente; menos de 7 no dependiente), encontramos que la NDSS-T y la escalas de impulso y de prioridad diferencian a los dependientes de no dependientes con el FTQ, con puntuaciones siempre mayores en los fumadores dependientes (38.61 vs. 29.40 en NDSS-T; 31.06 vs. 22.85 en la escala de impulso; 23.43 vs. 17.30 en la escala de prioridad). Con el SCID, la NDSS-T y las escalas de impulso, de prioridad, de continuidad y de estereotipia se diferencia a los fumadores dependientes de los no dependientes, siendo los dependientes los que tienen puntuaciones significativamente mayores en estas escalas, excepto en la escala de estereotipia en la que puntúan más los fumadores no dependientes (16.98 vs. 14.39) (tabla 8).

Tabla 6. Correlaciones entre la NDSS-T y sus escalas derivadas factorialmente con el Test de Fagerström (FTO), la SCID y el número de cigarrillos fumados diariamente.

\begin{tabular}{|l|c|c|c|c|c|}
\hline & $\begin{array}{c}\text { I. } \\
\text { Impulso }\end{array}$ & $\begin{array}{c}\text { II. } \\
\text { Prioridad }\end{array}$ & $\begin{array}{c}\text { III. } \\
\text { Continuidad }\end{array}$ & $\begin{array}{c}\text { IV. } \\
\text { Estereotipia }\end{array}$ & NDSS-T \\
\hline FOO & $.597^{* * *}$ & $.529^{* * *}$ & $.149^{*}$ & $-.146^{*}$ & $.537^{* * *}$ \\
\hline SCID & $.541^{* * *}$ & $.495^{* * *}$ & $.247^{* * *}$ & $-.178^{*}$ & $.522^{* * *}$ \\
\hline $\begin{array}{l}\text { No de cigarrillos } \\
\text { al dia }\end{array}$ & $.465^{* * *}$ & $.409^{* * *}$ & .066 & -.077 & $.420^{* * *}$ \\
\hline I. Impulso & 1.00 & $.900^{* * *}$ & $.333^{* * *}$ & $-.162^{*}$ & $.937^{* * *}$ \\
\hline II. Prioridad & $.900^{* * *}$ & 1.00 & $.239^{* * *}$ & -.029 & $.909^{* * *}$ \\
\hline III. Continuidad & $.333^{* * *}$ & $.239^{* * *}$ & 1.00 & $-.472^{* * *}$ & $.540^{* * *}$ \\
\hline IV. Estereotipia & $-.162^{*}$ & -.029 & $-.472^{* * *}$ & 1.00 & $-.143^{*}$ \\
\hline NDSS-T & $.937^{* * *}$ & $.909^{* * *}$ & $.540^{* * *}$ & $-.143^{*}$ & 1.00 \\
\hline
\end{tabular}

${ }^{*} p<.05 ;{ }^{* *} p<.01 ;{ }^{* * *} p<.001$

Tabla 7. Correlación entre los 19 ítems de la NDSS con las escalas de dependencia utilizadas y el número de cigarrillos fumados diariamente.

\begin{tabular}{|c|c|c|c|c|c|c|c|c|}
\hline & Total NDSS & I. Impulso & II. Prioridad & III. Continuidad & IV. Estereotipia & FTO & SCID & $\mathrm{N}^{\circ}$ cigarrillos dia \\
\hline 1. Irregular & .094 & .027 & -.050 & $.669^{* * *}$ & $-.576^{* * *}$ & -.009 & .063 & -.108 \\
\hline 2. Misma cantidad dia & .055 & .047 & .077 & -.007 & .138 & .079 & -.045 & -.025 \\
\hline 3. Viaje en avión & $.408^{* * *}$ & $.317^{* * *}$ & $.535^{* * *}$ & .015 & -.015 & $.223^{* *}$ & .095 & $.175^{*}$ \\
\hline 4. Amigos & $.399^{* * *}$ & $.301^{* * *}$ & $.521^{* * *}$ & .001 & .119 & $.214^{* *}$ & $.207^{* *}$ & $.235^{* * *}$ \\
\hline 5. Restaurante & $.645^{* * *}$ & $.617^{* * *}$ & $.694^{* * *}$ & $.214^{* *}$ & .060 & $.465^{* * *}$ & $.275^{* * *}$ & $.298^{* * *}$ \\
\hline 6. Fumo todo el dia & .104 & .133 & $.173^{*}$ & $-.278^{* * *}$ & $.726^{* * *}$ & .089 & -.062 & $.151^{*}$ \\
\hline 7. Situacional & $.501^{* * *}$ & $.333^{* * *}$ & $.255^{* * *}$ & $.735^{* * *}$ & $-.185^{* *}$ & $.146^{*}$ & $.284^{* * *}$ & .144 \\
\hline 8. Necesito fumar mas & $.663^{* * *}$ & $.733^{* * *}$ & $.476^{* * *}$ & $.297^{* * *}$ & -.132 & $.437^{* * *}$ & $.316^{* * *}$ & $.389^{* * *}$ \\
\hline 9. Fumo mas sin encontrarme mal & $.624^{* * *}$ & $.647^{* * *}$ & $.416^{* * *}$ & $.283^{* * *}$ & -.016 & $.378^{* * *}$ & $.369^{* * *}$ & $.151^{*}$ \\
\hline 10. Fumar para no sentirse mal & $.708^{* * *}$ & $.747^{* * *}$ & $.770^{* * *}$ & $.171^{*}$ & .078 & $.457^{* * *}$ & $.407^{* * *}$ & .144 \\
\hline 11. № cigarrillos varia & $.496^{* * *}$ & $.331^{* * *}$ & $.275^{* * *}$ & $.668^{* * *}$ & $-.294^{* * *}$ & $.169^{*}$ & .122 & $.389^{* * *}$ \\
\hline 12. Control sobre el tabaco & $-.429^{* * *}$ & $-.484^{* * *}$ & $-.482^{* * *}$ & -.120 & $.381^{* * *}$ & $-.369^{* * *}$ & $-.284^{* * *}$ & $.310^{* * *}$ \\
\hline 13. Consumo varía & $.484^{* * *}$ & $.283^{* * *}$ & $.225^{* *}$ & $.802^{* * *}$ & $-.275^{* * *}$ & .133 & $.251^{* * *}$ & $.401^{* * *}$ \\
\hline 14. Control externo & $.741^{* * *}$ & $.728^{* * *}$ & $.747^{* * *}$ & $.299^{* * *}$ & -.063 & $.307^{* * *}$ & $.458^{* * *}$ & .116 \\
\hline 15. Fumo lo mismo & $-.207^{* *}$ & $-.386^{* * *}$ & -.123 & $-.176^{*}$ & $.567^{* * *}$ & $-.320^{* * *}$ & $-.201^{* *}$ & $-.250^{* * *}$ \\
\hline 16. Ganas fuertes de fumar & $.774^{* * *}$ & $.795^{* * *}$ & $.820^{* * *}$ & $.219^{* *}$ & .009 & $.403^{* * *}$ & $.411^{* * *}$ & .053 \\
\hline 17. Regularidad & -.039 & -.015 & .041 & $-.301^{* * *}$ & $.783^{* * *}$ & -.053 & -.112 & -.079 \\
\hline 18. Alivio & $.766^{* * *}$ & $.789^{* * *}$ & $.845^{* * *}$ & $.180^{*}$ & .008 & $.401^{* * *}$ & $.502^{* * *}$ & $.310^{* * *}$ \\
\hline 19. Misma cantidad fin de semana & .049 & .077 & .101 & -.120 & $.495^{* * *}$ & .033 & .024 & -.022 \\
\hline
\end{tabular}

${ }^{*} p<.05 ;{ }^{* *} p<.01 ;{ }^{* * *} p<.001$ 
Tabla 8. Dependencia de la nicotina según el Test de Fagerström (FTQ) y la SCID en relación al NDSS-T y sus escalas.

\begin{tabular}{|c|c|c|c|c|c|c|c|}
\hline & & \multicolumn{3}{|c|}{ Test de Fagerström (FTQ) } & \multicolumn{3}{|c|}{ SCID } \\
\hline & & No dependiente $(p<7)$ & Dependiente $(p \geq 7)$ & $\mathrm{t}$ & No dependiente & Dependiente & $\mathrm{T}$ \\
\hline \multirow{2}{*}{ I. Impulso } & Media & 22.85 & 31.06 & \multirow{2}{*}{$-8.109^{* * *}$} & 19.86 & 28.64 & \multirow{2}{*}{$-7.162^{* * *}$} \\
\hline & D.T. & 7.19 & 6.33 & & 6.08 & 7.29 & \\
\hline \multirow{2}{*}{ II. Prioridad } & Media & 17.30 & 23.43 & \multirow{2}{*}{$-6.959^{* * *}$} & 15.40 & 21.52 & \multirow{2}{*}{$-6.555^{* * *}$} \\
\hline & D.T. & 5.79 & 6.11 & & 4.97 & 6.47 & \\
\hline \multirow{2}{*}{ III. Continuidad } & Media & 10.50 & 11.42 & \multirow{2}{*}{-1.624} & 9.40 & 11.39 & \multirow{2}{*}{$-3.039^{* *}$} \\
\hline & D.T. & 3.91 & 3.71 & & 4.09 & 3.65 & \\
\hline \multirow{2}{*}{ IV. Estereotipia } & Media & 15.46 & 14.45 & \multirow{2}{*}{1.377} & 16.98 & 14.39 & \multirow{2}{*}{$3.047^{* *}$} \\
\hline & D.T. & 5.03 & 4.88 & & 4.71 & 4.91 & \\
\hline \multirow{2}{*}{ NDSS-T } & Media & 29.40 & 38.61 & \multirow{2}{*}{$-7.140^{* * *}$} & 25.91 & 35.94 & \multirow{2}{*}{$-6.491^{* * *}$} \\
\hline & D.T. & 8.61 & 8.78 & & 7.53 & 9.23 & \\
\hline
\end{tabular}

${ }^{*} p<.05 ;{ }^{* *} p<.01 ; i^{* * *} p<.001$

Tabla 9. Diferencias en la NDSS-T y sus escalas en relación al consumo de cigarrillos (1-19, 20 o más).

\begin{tabular}{|c|c|c|c|c|}
\hline & & \multicolumn{3}{|c|}{ Consumo diario de cigarrillos } \\
\hline & & $1-19$ & 20 o más & $\mathrm{t}$ \\
\hline \multirow{2}{*}{ I. Impulso } & Media & 21.36 & 27.85 & \multirow{2}{*}{$-4.636^{* * *}$} \\
\hline & D.T. & 6.62 & 7.73 & \\
\hline \multirow{2}{*}{ II. Prioridad } & Media & 16.89 & 20.86 & \multirow{2}{*}{$-3.293^{* *}$} \\
\hline & D.T. & 5.75 & 6.66 & \\
\hline \multirow{2}{*}{ III. Continuidad } & Media & 10.14 & 11.11 & \multirow{2}{*}{-1.361} \\
\hline & D.T. & 3.88 & 3.82 & \\
\hline \multirow{2}{*}{ IV. Estereotipia } & Media & 15.06 & 14.99 & \multirow{2}{*}{.075} \\
\hline & D.T. & 4.06 & 5.19 & \\
\hline \multirow{2}{*}{ NDSS-T } & Media & 27.75 & 35.01 & \multirow{2}{*}{$-4.151^{* * *}$} \\
\hline & D.T. & 7.98 & 9.71 & \\
\hline
\end{tabular}

${ }^{*} p<.05 ;{ }^{* *} p<.01 ; * * * p<.001$

\section{Características de los fumadores según la evaluación de la NDSS}

El análisis de distintas variables demográficas y de consumo de cigarrillos con la NDSS-T nos indica resultados en la línea de lo que sería esperable. Así, hay diferencias significativas en el factor prioridad. Considerando los solteros (n $=80$ ), casados ( $n=71$ ) y otros ( $n=32$, separados, divorciados y viudos) encontramos que hay diferencias significativas entre solteros y casados en prioridad, en el que puntúan más los fumadores solteros que los casados (21.13 vs. 18.42). No aparecen otras diferencias en el resto de las escalas. Tampoco aparecen diferencias por género ni por edad, con la excepción de la escala NDSS-T en donde puntúan más los más jóvenes ( 35.31 vs. $32.28, t=2.00, p<.05$ ).

En relación al consumo de cigarrillos, al diferenciar a los fumadores en consumidores de 1-19 cigarrillos o de 20 o más al dia, encontramos diferencias significativas en la escala NDSS-T y en dos de las cuatro escalas factoriales (impulso y prioridad). En todos los casos puntúan siempre más alto los que fuman más cigarrillos diariamente (tabla 9).
Predicción de la dependencia de la nicotina (SCID) con la NDSS utilizando curvas ROC

Las curvas ROC (curva Característica de Operación del Receptor) es un método gráfico que permite determinar el punto óptimo de clasificación a partir de las funciones de diferenciación entre dos grupos. Ofrece una representación gráfica de la sensibilidad frente a la especificidad de una herramienta, así como estadísticos asociados a las características de la herramienta de clasificación analizada (Pardo y Ruiz, 2002). Las curvas ROC permiten determinar el punto de corte óptimo en la asignación de casos a grupos. Con este procedimiento valoramos el comportamiento de una función discriminante entre grupos, tal como la generada por el análisis discriminante o el análisis de regresión logística, proporcionándonos un gráfico de la curva ROC y estimando el área existente bajo la curva, considerándose habitualmente que es significativa si está por encima de 0.5 del área verdadera. La gráfica nos proporciona en el eje de abscisas la especificidad (proporción de falsos positivos) y en el eje de ordenadas la sensibilidad (proporción de aciertos positivos). El valor que nos da del área puede ir de 0 a 1 . Cuanto más se acerque a 1 (en caso de ser 1 tendríamos un 100\% de aciertos positivos y $0 \%$ de falsos positivos) mayor sensibilidad y menor especificidad. Esta sería una curva perfecta. Una mala curva es cuando el valor es menor de 0.5 (50\% de aciertos positivos y $50 \%$ de falsos positivos) o se acerca a 0 (todos falsos positivos). Normalmente se consideran adecuadas curvas ROC cuando tienen un área de 0.5 o mayor.

En nuestro caso podemos utilizar curvas ROC al tener como variable predictiva o criterio la dependencia tal como la evalúa la SCID (3 o más ítems positivos para dependencia) respecto a la no dependencia (de 0 a 2 ítems positivos) y como predictoras las cuatro escalas de la NDSS y, aparte, la escala NDSS-T.

Los resultados indican que 140 fumadores de la presente muestra tienen dependencia de la nicotina (76.5\%) y 43 no (23.5\%) al ser evaluados con la SCID. Con las distintas escalas de la NDSS y su puntuación total (NDSS-T) tres de 
las cuatro escalas (excepto el factor 4 de estereotipia) tienen una puntuación bajo el área superior a 0.50 , con valores tan altos como 0.80 para NDSS-T y el primer factor de impulso con 0.82 (figura 2). Por tanto, excepto para el factor 4 , que no es predicho adecuadamente, en los demás la predicción es significativa en todos los casos.

Si comparamos el funcionamiento de la escala NDSS-T con el FTO nos encontramos que ambas predicen adecuadamente la dependencia, aunque con un mejor nivel de ajuste la escala NDSS-T que tiene una puntuación de 0.80 bajo la curva y el FTO de 0.70 . Esto indica claramente que ambas discriminan bien a los fumadores dependientes de los no dependientes aunque la NDSS-T ajusta un poco mejor dicha predicción.

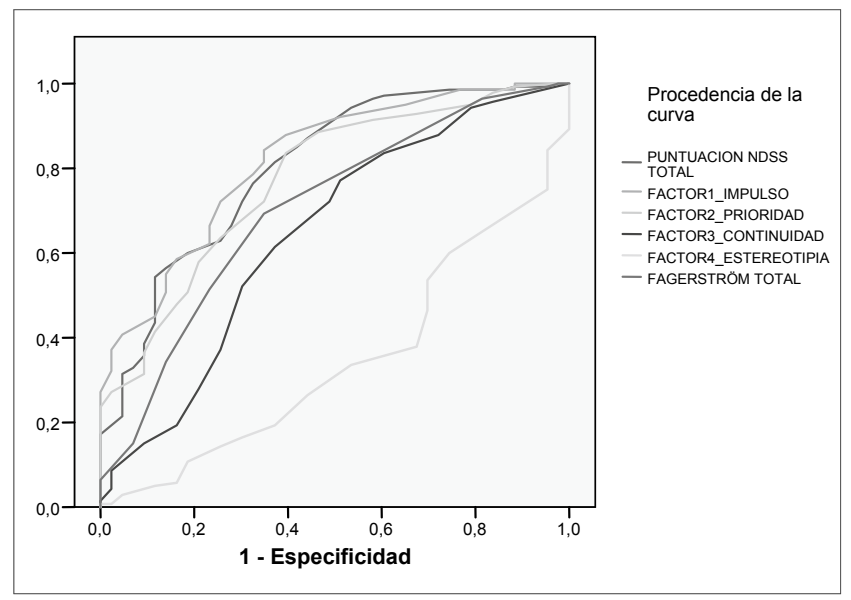

Figura 2. Curva ROC de la NDSS-T y sus escalas. Tests de Fagerström (FTO) y SCID.

\section{Predicción de la dependencia en función de las variables del estudio}

Un análisis de regresión step-wise en el que hemos incluido los 4 factores de la NDSS, su puntuación total, el FTO y el número de cigarrillos diarios (todas variables continuas) para predecir el número de síntomas en la SCID nos indica que la única variable significativa es el primer factor de la NDSS (impulso) $(R=0.541)$. Si utilizamos el número de cigarrillos diarios, FTO y NDSS-T entonces la única variable que aparece significativa es la NDSS-T $(R=0.522)$.

Si utilizamos un análisis de regresión logística (hacia adelante condicional) para dependiente vs. no dependiente según la SCID con las mismas variables anteriores (cuatro escalas de la NDSS, NDSS-T, FTO y número de cigarrillos diarios), entonces la única escala de la NDSS que aparece como significativa es la escala I. Impulso (Wald $=30.38, p<.001$ ). Si eliminamos las cuatro escalas y dejamos las otras variables anteriores entonces la única que surge como significativa es la NDSS-T (Wald = 27.57, p <.001), mostrándose claramente en éste como en los casos anteriores la utilidad de la escala ya que el modelo permite clasificar correctamente el $80.9 \%$ y el $83.6 \%$ de los fumadores en uno y otro caso.

Por tanto, los resultados anteriores indican la utilidad de la NDSS ya que su nivel de predicción de la dependencia de la nicotina es alto y a veces mejor que el de otros criterios como el número de cigarrillos diarios y la escala FTO.

\section{DISCUSIÓN}

Los resultados del presente estudio indican que la NDSST en esta muestra de personas que demandan tratamiento fundamentalmente por problemas de abuso o dependencia del alcohol, presenta unas propiedades psicométricas satisfactorias. Así, el coeficiente alfa de Cronbach, como indicador de la consistencia interna, de la subescala general de dependencia (el primer factor sin rotar resultante del análisis factorial y consistente en 13 de los 19 items de la NDSS), presenta un valor satisfactorio de 0.80 . Igualmente surgen cuatro de los cinco primeros factores que se han propuesto en la validación original de Shiffman et al. (2004), los de impulso, prioridad, continuidad y estereotipia. No aparece el quinto propuesto de tolerancia. Los dos primeros factores, impulso y prioridad, tienen buena fiabilidad ( 0.84 cada uno), mientras que la de continuidad y estereotipia es moderada (0.68 y 0.63 , respectivamente). Un resultado semejante obtuvieron Piper et al. (2008) al utilizar tres muestras distintas de fumadores de cigarrillos (10 o más cigarrillos diarios) y los de Okuyemi et al. (2007) con fumadores de pocos cigarrillos, aunque con menor nivel de fiabilidad que los obtenidos por Shiffman et al. (2004). Sin embargo, en todos los estudios citados, incluyendo el nuestro, la fiabilidad de la escala total es buena, lo que indica que la puntuación total evalúa adecuadamente y de manera fiable el factor general de dependencia de la nicotina.

El análisis de la escala total y de los cuatro factores de la NDSS indica la utilidad discriminativa de la escala total y de los dos primeros factores de impulsividad y prioridad en casi todas las comparaciones, como ocurre en función del consumo de cigarrillos diario y la dependencia de la nicotina mediante el FTO. Donde aparecen diferencias en todas las escalas de la NDSS y en su escala total es en la diferenciación con el SCID de los fumadores dependientes de los no dependientes. Aquí los fumadores dependientes tienen mayores puntuaciones en la escala total, en la de impulso, prioridad y continuidad, aunque menor en la de estereotipia. Estos resultados indican que la evaluación clínica de dependencia de la nicotina, como se ha hecho en este estudio con criterios DSM-IV-TR mediante la SCID discrimina mejor todas las escalas que forman la NDSS a diferencia de otras variables habitualmente utilizadas como son el número de cigarrillos fumados a diario o el FTO.

Con los criterios de la SCID el $76.50 \%$ de los fumadores de la presente muestra son dependientes de la nicotina lo que supone un porcentaje elevado. Los estudios que se han realizado sobre esta cuestión han indicado distintos porcentajes de dependencia de la nicotina, que depende fundamentalmente del tipo de muestra utilizada. Consideramos que por el tipo de muestra utilizada en este estudio, fumadores que acuden a una Unidad de Alcoholismo demandando tratamiento para su abuso o dependencia del alcohol, y por la relación que existe de un mayor consumo de tabaco en este tipo de personas (Nieva et al., 2004), los resultados obtenidos son coherentes y esperables. Así, estos resultados son semejantes a los de Hughes, Olivito, Riggs, Kenny, Kiguori, Pillitteri, et al. (2004), que encontraron un $63 \%$ y $86 \%$ en 
dos estudios distintos, aunque ligeramente mayores a los obtenidos en otros estudios epidemiológicos, como los de Breslau, Jhonson, Hiripi y Kessler (2001) y Donny y Dierker (2007), en donde encontraron un $62.8 \%$ de dependientes a los 45-54 años y 60.6\%, respectivamente.

Para comprobar la utilidad de la escala NDSS al disponer de un criterio de dependencia como es la SCID, hemos utilizado curvas ROC para ver la capacidad predictiva de la NDSS respecto a la dependencia o no de la nicotina. Las curvas ROC nos indican que tanto la escala total de la NDSS como los tres primeros factores tienen muy buen funcionamiento, estando las puntuaciones bajo el área entre 0.64 y 0.80 . En cambio para la escala de estereotipia la discriminación es baja, de solo un 0.34 bajo la curva.

Si utilizamos la NDSS-T y el FTO en relación con la dependencia evaluada con la SCID surge que la predicción con la NDSS-T es excelente, con un valor de 0.80 bajo la curva, siendo también buena con el FTO que tiene un 0.70 bajo la curva. Esto indica que tanto la NDSS-T como el FTO evalúan una parte importante de la varianza de la dependencia de la nicotina tal como la evalúa lal SCID. También tienen un buen nivel de predicción las dos primeras escalas derivadas factorialmente (impulso, 0.81; prioridad, 0.77), adecuado con la tercera (continuidad, 0.64), pero baja con la de estereotipia (0.34).

Otro resultado destacable en este estudio es la capacidad predictiva de la NDSS-T en relación con otros predictores clásicamente utilizados para conocer el nivel de dependencia de la nicotina o el nivel de implicación en la conducta de fumar. El análisis de regresión múltiple realizado en el que hemos utilizado los 4 factores de la NDSS, la NDSS-T, el FTO y el número de cigarrillos diarios, indica que la única variable que resulta significativa es el primer factor de la NDSS, el de de impulso. Si utilizamos sólo la NDSS-T sin sus escalas junto con las mismas variables anteriores entonces la única variable que aparece significativa para predecir la dependencia es la NDSS-T. Por tanto, estos resultados indican la utilidad de la NDSS ya que su nivel de predicción de la dependencia de la nicotina es alto y a veces incluso mejor que el de otros criterios como el número de cigarrillos diarios o el FTQ.

El análisis de correlaciones entre las distintas variables del estudio nos indica que la correlación entre la NDSS-T y otras escalas o variables relacionadas con el consumo de tabaco son altas y siempre significativas, siendo las $r$ de 0.54 $(p<.001)$ con el FTQ y de $0.52(p<.001)$ con la SCID. También la correlación entre la NDSS-T y el número de cigarrillos fumados diariamente es alta, de 0.42 ( $p<.001)$. Estos resultados indican que tanto las distintas escalas, como el consumo de cigarrillos, tienen importantes elementos en común y la NDSS-T se relaciona bien con todos ellos. Quizás el dato más importante es que la correlación con la SCID es equivalente con la NDSS-T y con el FTQ.

La principal conclusión de este estudio es que la NDSS-T en esta muestra de fumadores que demandan tratamiento por sus problemas de abuso o dependencia del alcohol tiene un adecuado funcionamiento para su escala total, como para tres de sus cuatro escalas derivadas factorialmente, con un adecuado poder discriminativo para las variables más relevantes que suelen considerarse en esta dependencia (ej., número de cigarrillos diarios). Los 13 ítems que componen la escala total la convierten en un instrumento breve y útil para evaluar la dependencia de la nicotina y en una escala más para poder utilizar con nuestros fumadores.

Dice Kassel (2000) que "la operacionalización del constructo de dependencia de la nicotina es todavía un trabajo en progreso $y$, como tal, no hay buenas medidas estándar con respecto a su evaluación" (p. 32). Este estudio muestra que esto es cierto. Necesitamos conocer mejor la dependencia de la nicotina, tener adecuados instrumentos de evaluación, a poder ser breves (de León, Díaz, Becoña, Gurpegui, Jurado y González-Pinto, 2003) y ver la capacidad predictiva de los mismos. De ahí que todavía tengamos que seguir avanzando en este tema, tanto a nivel teórico como de desarrollo de nuevos instrumentos de evaluación (Brandon, Herzog, Irwin y Gwaltney, 2004).

Las principales limitaciones del presente estudio son, en primer lugar, el tipo de muestra, que aunque es una muestra clínica siempre es deseable que sea mayor. Con todo, la misma representa a un tipo de personas fumadoras de gran relevancia para poder intervenir en ellas y que suelen tener una alta prevalencia de consumo de tabaco. Una segunda limitación está en que lo ideal sería utilizar un mayor número de medidas que evaluasen la dependencia de la nicotina. Lo hemos intentado solventar en este estudio utilizando varias de las que hoy se consideran más relevantes en los estudios sobre dependencia de la nicotina. Y, una última limitación hace referencia a la necesidad de disponer de más información sobre la historia de fumar de los fumadores.

\section{REFERENCIAS}

American Psychiatric Association (2000). Diagnostic and statistical manual for mental disorders, 4th ed., revised text. Washington, D.C.: American Psychiatric Association (trad. cast.: Barcelona, Masson, 2002).

Ballbé, M., Mondón, S., Nieva, G., Walter, M., Saltó, E. y Gual, A. (2008). Evaluación de un programa de formación de profesionales sanitarios sobre abordaje del tabaquismo en pacientes hospitalizados. Adicciones, 20, 125-130.

Becoña, E. (2006). Tabaco. Prevención y tratamiento. Madrid: Pirámide. Becoña, E., Gómez-Durán, B., Alvarez-Soto, E. y García, M. P. (1992). Scores of Spanish smokers on the Fagerström's Tolerance Questionnaire. Psychological Reports, 71, 1227-1233.

Becoña, E., López, A., Fernández del Río, E. y Míguez, M. C. (2008). Evaluación de la dependencia de la nicotina con criterios DSM-IV a través de la SCID. Santiago de Compostela: Unidad de Tabaquismo, Universidad de Santiago de Compostela.

Becoña, E. y Lorenzo, M. C. (2004). Evaluación de la conducta de fumar. Adicciones, 16 (Supl. 2), 201-226.

Becoña, E. y Vázquez, F. (1998). The Fagerström Test for Nicotine Dependence in a Spanish sample. Psychological Reports, 83, 1455-1458. 
Brandon, T. H., Herzog, T. A., Irvin, J. E. y Gwaltney, C. J. (2004). Cognitive and social learning models of drug dependence: Implications for the assessment of tobacco dependence in adolescents. Addiction, 99 (Supl. 1), 51-77.

Breslau, N., Johnson, E. O., Hiripi, E. y Kessler, R. (2001). Nicotine dependence in the United States. Prevalence, trends, and smoking persistence. Archives of General Psychiatry, 58, 810-816.

Brislin, R. W., Lonner, W. J. y Thorndike, E. M. (1973). Cross-cultural research methods. Nueva York: Wiley.

Broms, U., Madden, P. A. F., Health, A. C., Pergadia, M. L., Shiffman, S. y Kaprio, J. (2007). The Nicotine Dependence Syndrome Scale in Finnish smokers. Drug and Alcohol Dependence, 89, 42-51.

Carballo, J. L., Fernández-Hermida, J. R., Secades-Villa, R. y GarciaRodríguez, O. (2008). Determinantes de la recuperación de los problemas de alcohol en sujetos tratados y no tratados en una muestra española. Adicciones, 20, 49-58.

Clark, D. B., Wood, D. S., Martin, C. S., Cornelius, J. R., Lynch, K. G. y Shiffman, S. (2005). Multidimensional assessment of nicotine dependence in adolescents. Drug and Alcohol Dependence, 77, 235-242.

Costello, D., Dierker, L., Sledjeski, E., Flaherty, B., Flay, B. y Shiffman, S. (2007). Confirmatory factor analysis of the Nicotine Dependence Syndrome Scale in an American college sample of light smokers. Nicotine \& Tobacco Research, 9, 811-819.

Courvoisier, D. y Etter, J-F. (2008). Using item response theory to study the convergent and discriminant validity of three questionnaires measuring cigarettes dependence. Psychology of Addictive Behaviors, 22, 391-401.

de Leon, J., Becoña, E., Gurpegui, M., González-Pinto, A. y Díaz, F. J. (2002). The association between high nicotine dependence and severe mental illness may be consistent across countries. Journal of Clinical Psychiatry, 63, 812-816.

de Leon, J., Diaz, F. J., Becoña, E., Gurpegui, M., Jurado, D. y GonzálezPinto, A. (2003). Exploring brief measures of nicotine dependence for epidemiological surveys. Addictive Behaviors, 28, 1481-1486.

Dierker, L. C., Donny, E., Tiffany, S., Colby, S. M., Perrine, N. y Clayton, R. R. (2007). The association between cigarette smoking and DSMIV nicotine dependence among first year college students. Drug and Alcohol Dependence, 86, 106-114.

Donny, E. C. y Dierker, L. C. (2007). The absence of DSM-IV nicotine dependence in moderate-to-heavy daily smokers. Drug and Alcohol Dependence, 89, 93-96.

Edwards, G. (1986). The alcohol dependence syndrome: A concept as stimulus to enquiry. British Journal of Addiction, 81, 171-183.

Fagerström, K. O. (1978). Measuring degree of physical dependence on tobacco smoking with reference to individualization of treatment. Addictive Behaviors, 4, 235-241.

Fagerström, K. O. y Schneider, N. G. (1989). Measuring nicotine dependence: A review of Fagerström Tolerance Questionnaire. Journal of Behavioral Medicine, 12, 159-182.

Fiore M. C., Jaén, C. R., Baker, T. B., Balley, W. W., Benowitz, N. L., Curry, S. L., et al. (2008). Treating tobacco use and dependence: 2008 update. Rockville, MD: U. S. Department of Health and Human Services, Public Health Service.

First, M. B., Spitzer, R. L., Gibbson, M., Williams, J. B.W. y SmithBenjamin, L. (1998). Guía del usuario de la entrevista clínica estructurada para los trastornos del eje I del DSM-IV- SCID-I. Barcelona: Masson.

Heatherton, T. F., Kozlowski, L. T., Frecker, R. C. y Fagerström, K. 0. (1991). The Fagerström Test for Nicotine Dependence: A revision of the Fagerström Tolerance Questionnaire. British Journal of Addictions, 85, 1119-1127.

Hughes, J. R., Oliveto, A. H., Riggs, R., Kenny, M., Kiguori, A., Pillitteri, J. L. y MacLaughlin, M. A. (2004). Concordance of different measures of nicotine dependence: Two pilot studies. Addictive Behaviors, 29, 1527-1539.

Kassel, J. D. (2000). Are adolescent smokers addicted to nicotine? The suitability of the nicotine dependence construct as applied to adolescents. Journal of Child and Adolescent Substance Abuse, 9, 27-49.

Kline, P. (1994). An easy guide to factor analysis. Londres: Routledge.

López, A., Becoña, E., Lloves, M., Moneo, A., Vieitez, I., Cancelo, et al. (2007). Evolución del tratamiento y consumo en personas con dependencia de la cocaina a los doce meses, en función de la psicopatología asociada y el funcionamiento cotidiano. Adicciones, 19, 251-265.

Moolchan, E. T., Radzius, A., Epstein, D. H., Uhl, G., Gorelick, D. A., Cdet, J. L., et al. (2002). The Fagerström Test for Nicotine Dependence and the Diagnostic Interview Schedule - Do they diagnose the same smokers? Addictive Behaviors, 27, 101-113.

Nieva, G., Gual, A., Ortega, Ll. y Mondón, S. (2004). Alcohol y tabaco. Adicciones, 16 (Supl. 2), 191-199.

Nieva, G. y Gual, A. (2005). El tratamiento del tabaquismo en alcohólicos: ¿miopía o negligencia? Adicciones, 17, 177-180.

Okuyemi, K. S., Pulvers, K. M., Cox, L. S., Thomas, J. L., Kaur, H., Mayo, M. S., et al. (2007). Nicotine dependence among African American light smokers: A comparison of three scales. Addictive Behaviors, 32, 1989-2002.

Organización Mundial de la Salud (1992). CIE-10. Clasificación de los trastornos mentales y del comportamiento. Madrid: Meditor.

Pardo, A. y Ruiz, M. A. (2002). SPSS 11. Guía para el análisis de datos. Madrid: McGraw-Hill.

Pereiro, C., Becoña, E., Córdoba, R., Martínez, J. y Pinet, C. (2008). Tabaquismo. Guías clínicas Socidrogalcohol basadas en la evidencia cientifica. Barcelona: Socidrogalcohol.

Pomerleau, C. S., Carton, S. M., Lutzke, M. L., Flessland, K. A. y Pomerleau, O. F. (1994). Reliability of the Fagerström Tolerance Questionnaire and the Fagerström Test for Nicotine Dependence. Addictive Behaviors, 19, 33-39.

Piper, M. E., McCarthy, D. E., Bolt, D. M., Smith, S. S., Lerman, C., Bnowith, N., et al. (2008). Assessing dimensions of nicotine dependence: An evaluation of the Nicotine Dependence Syndrome Scale (NDSS) and the Wisconsin Inventory of Smoking Dependence Motives (WISDM). Nicotine \& Tobacco Research, 6, 1009-1020.

Shiffman, S. y Paton, S. M. (1999). Individual differences in smoking: Gender and nicotine addiction. Nicotine \&t Tobacco Research, 1, 153-157.

Shiffman, S. y Sayette, M. A. (2005). Validation of the Nicotine Dependence Syndrome Scale (NDSS): a criterion-group design contrasting chippers and regular smokers. Drug and Alcohol Dependence, 79, 45-52.

Shiffman, S., Waters, A. J. y Hickcox, M. (2004). The Nicotine Dependence Syndrome Scale: a multidimensional measure of nicotine dependence. Nicotine \& Tobacco Research, 6, 327-348.

Shadel, W. G., Shiffman, S., Niaura, R., Nichter, M. y Abrams, D. B. (2000). Current models of nicotine dependence: What is known and what is needed to advance understanding of tobacco etiology among youth. Drug and Alcohol Dependence, 59 (Supl. 1), S9-S21. 
Sledjeski, E. M., Dierker, L. C., Costello, D., Shiffman, S., Donny, E. y Flay, B. R. (2007). Predictive utility of four nicotine dependence measures in a college sample. Drug and Alcohol Dependence, 87, 10-19.

Sterling, K. L., Mermelstein, R., Turner, L., Diviak, K.,Flay, B. y Shiffman, S. (2008). The Nicotine Dependence Syndrome Scale: Factorial and predictive validity among youth smoking samples. Manuscrito remitido para publicación.

U. S. D. H. H. S. (1988). The health consequences of smoking. Nicotine addiction. A report of the Surgeon General. Rockville, MD: U.S. Department of Health and Human Services.
U. S. D. H. H. S. (2000). Reducing tobacco use: A report of the Surgeon General. Atlanta, GA: U.S. Department of Health and Human Services, Centers for Disease Control and Prevention, National Center for Chronic Disease Prevention and Health Promotion, Office on Smoking and Health.

U. S. D. H. H. S. (2004). The health consequences of smoking: A report of the Surgeon General. Atlanta, GA: U. S. Department of Health and Human Services, Centers for Disease Control and Prevention, National Center for Chronic Disease Prevention and Health Promotion, Office on Smoking and Health. 
